\title{
THÉORÈMES LIMITES POUR CERTAINES FONCTIONNELLES ASSOCIÉES AUX PROCESSUS STABLES SUR L'ESPACE DE HÖLDER
}

\author{
M. Ait Ouahra et M. Eddahbi
}

Abstract

In this paper we study the Hölder regularity property of the local time of a symmetric stable process of index $1<\alpha \leq 2$ and of its fractional derivative as a doubly indexed process with respect to the space and the time variables. As an application we establish some limit theorems for occupation times of one-dimensional symmetric stable processes in the space of Hölder continuous functions. Our results generalize those obtained by Fitzsimmons and Getoor for stable processes in the space on continuous functions. The limiting processes are fractional derivatives and Hilbert transforms of local times.

\section{Introduction}

Soit $X=\left\{X_{t}: t \geq 0\right\}$ un processus stable symétrique d'indice $1<$ $\alpha \leq 2$ à valeurs réelles avec $X_{0}=0$ i.e. un processus càdlàg à accroissement indépendant stationnaire d'exposant $\Psi$, défini par:

$$
\mathbb{E} \exp \left(i \lambda X_{t}\right)=\exp (-t \Psi(\lambda)), \quad t \geq 0, \lambda \in \mathbb{R}
$$

avec $\Psi(\lambda)=|\lambda|^{\alpha}$.

Pour tout $t>0$ on définit la mesure aléatoire $\mu_{t}(\cdot)$ par $\mu_{t}(A)=$ $\int_{0}^{t} \mathbb{1}_{A}\left(X_{s}\right) d s$, (où $A \subset \mathbb{R}$ est un borélien de $\mathbb{R}$ et $\mathbb{1}_{A}(\cdot)$ est la fonction indicatrice de $A) \mu_{t}(A)$ est la mesure d'occupation de $X$ dans le Borélien $A$. Il est bien connu d'après $[\mathbf{8}],[\mathbf{5}]$ et $[\mathbf{1}]$ que la mesure $\mu_{t}(A)$ admet une densité notée $L_{t}^{x}$ par rapport à la mesure de Lebesgue, $\left(L_{t}^{x}: t \geq 0, x \in \mathbb{R}\right)$ est appelé la famille des temps locaux associée à $X$, de plus $L_{t}^{x}$ admet

2000 Mathematics Subject Classification. 60F25, 60G52.

Mots-clés. Processus stables, temps local, transformé de Hilbert, dérivée fractionnaire, fonctionnels additives, norme de Hölder.

Cette note a été complétée au moment où le second auteur était en visite au Centre de Recerca Matemàtica (CRM) Barcelona, Spain. 
une version p.s. continue (en $t$ et $x$ ) et $L_{t}^{x}$ vérifie la formule de densité d'occupation et la propriété de scaling suivantes:

$$
\int_{0}^{t} f\left(X_{s}\right) d s=\int_{\mathbb{R}} f(x) L_{t}^{x} d x \quad \forall f \text { borélienne bornée }
$$

$$
\left\{L_{\lambda t}^{\lambda \frac{1}{\alpha} x}: t \geq 0\right\} \stackrel{\mathcal{L}}{=}\left\{\lambda^{1-\frac{1}{\alpha}} L_{t}^{x}: t \geq 0\right\} \text { pour tout } \lambda>0 .
$$

Depuis que Trotter [23] a démontré l'existence d'une version bi-continue du processus du temps local du mouvement brownien uni-dimensionnel, on ne cesse de découvrir des propriétés profondes des temps locaux, et on a abouti surtout à une belle théorie en ce qui concerne le temps local du brownien. L'étude des temps locaux est motivée par le rôle prépondérant du temps local dans la théorie des excursions. D'autre part l'utilisation des temps locaux facilite souvent l'étude des fonctionnelles additives d'un processus de Markov.

Le temps local d'un processus stable est une fonctionnelle additive particulière associée à ce processus, elle appartient à la classe des fonctionnelles additives continues, associées aux processus stables, d'énergie nulle, au sens de [11]. Cette classe contient des exemples importants notamment la dérivée fractionnaire et la transformée de Hilbert du temps local. Elles ont été étudiées, dans le cas du mouvement Brownien, par plusieurs auteurs dans divers points de vu. L'existence de la valeur principale de Cauchy du temps local a été remarquée par Itô et McKean [14]. Yor [28], Yamada [24], Nakao [20] et Bertoin [2] ont développés l'étude de ces fonctionnelles, ce qui a permis par exemple d'obtenir une généralisation de la formule de Itô (cf. [28] et [3]) et d'établir des théorèmes limites pour les temps d'occupations du mouvement Brownien (cf. $[\mathbf{2 6}]$ ).

Les théorèmes limites pour les mesures d'occupations ont été étudiés par Yamada $[\mathbf{2 6}],[\mathbf{2 7}]$ dans le cas $\alpha=2$ (i.e. $X$ est un mouvement brownien), voir aussi $[\mathbf{1 5}],[\mathbf{1 6}]$ et par [10] pour les processus stables. Les convergences dans tous ces théorèmes sont étudiés dans le cas des trajectoires continues et notre but dans cette note est de démontrer qu'on peut étendre les résultats de Yamada [26] et Fitzsimmons et Getoor [10] sur les théorèmes limites pour les mesures d'occupations associés aux processus stables uni-dimensionnel à la topologie des espaces de Hölder.

\subsection{Espaces fonctionnelles.}

Nous considérons les espaces de fonctions vérifiant une condition de Hölder en norme uniforme. Soit $f \in \mathcal{C}_{0}([0,1])$, le module de continuité 
de $f$ en norme uniforme, notée $\omega_{\delta}(f, \eta)$, est défini par

$$
\omega_{\delta}(f, \eta)=\sup _{0<|t-s| \leq \eta} \frac{|f(t)-f(s)|}{|t-s|^{\delta}} .
$$

Pour $0<\delta<1$, on définit

$$
\mathcal{C}_{0}^{\delta}:=\left\{f:[0,1] \longrightarrow \mathbb{R}: \text { tel que } f(0)=0 \text { et } \forall \eta>0, \omega_{\delta}(f, \eta)<+\infty\right\} .
$$

Il est bien connu que $\mathcal{C}_{0}^{\delta}$, muni de la norme $\|f\|_{\delta}:=\omega_{\delta}(f, 1)$, est un espace de Banach.

Remarque 1. $\mathcal{C}_{0}^{\delta}$ n'est pas séparable, à cause de ce désavantage on introduit un sous espace fermé séparable $\mathcal{C}_{0}^{\delta, 0}$, défini par:

$$
\mathcal{C}_{0}^{\delta, 0}:=\left\{f \in \mathcal{C}_{0}^{\delta} \text { tel que } \lim _{\eta \rightarrow 0} \omega_{\delta}(f, \eta)=0\right\} .
$$

Dans ce qui suit $C$ désignera une constante qui change de valeur d'une ligne à l'autre.

\section{Temps local et dérivées fractionnaires}

\subsection{Régularité du temps local.}

Soit $X=\left\{X_{t}: t \geq 0\right\}$ un processus stable symétrique d'indice $1<$ $\alpha \leq 2$ à valeurs dans $\mathbb{R}$ et soit $\left\{L_{t}^{x}:(t, x) \in \mathbb{R}^{+} \times \mathbb{R}\right\}$ son temps local. D'après [19] pour tout $T>0$ les conditions suivantes sont vérifiées presque sûrement:

$\forall 0<\beta<\frac{\alpha-1}{\alpha}, \exists C>0$ telle que $\forall 0 \leq t, s \leq T,|x| \leq M$

$$
\left|L_{t}^{x}-L_{s}^{x}\right| \leq C|t-s|^{\beta},
$$

$\forall 0<\beta<\frac{\alpha-1}{2}, \exists C>0$ telle que $\forall 0 \leq t \leq T,|x|,|y| \leq M$

$$
\left|L_{t}^{x}-L_{t}^{y}\right| \leq C|x-y|^{\beta} .
$$

Remarque 2. Bien que le processus stable $\left\{X_{t}: t \geq 0\right\}$ n'est pas nécessairement continu, son temps local possède une version jointement hölderienne en $t$ et $x$. 


\subsection{Dérivées fractionnaires.}

Soit $\beta>0$, considérons l'espace $\mathcal{C}^{\beta}$ définit par

$$
\begin{array}{r}
\mathcal{C}^{\beta}:=\left\{f: \mathbb{R} \longrightarrow \mathbb{R}: \text { tel que }|f(x)-f(y)| \leq C(f, \beta)|x-y|^{\beta},\right. \\
\forall|x|,|y| \leq M\} .
\end{array}
$$

Pour $\gamma \in] 0, \beta[$ on définit la dérivée fractionnaire d'ordre $\gamma$ d'une fonction $f$ appartenant à $\mathcal{C}^{\beta} \cap \mathrm{L}^{1}(\mathbb{R})$ par:

$$
D_{ \pm}^{\gamma} f(x)=\frac{1}{\Gamma(-\gamma)} \int_{0}^{+\infty} \frac{f(x \pm y)-f(x)}{y^{1+\gamma}} d y
$$

et on défnit l'opérateur $D^{\gamma}$ par $D^{\gamma}:=D_{+}^{\gamma}-D_{-}^{\gamma}$.

Puisque $\frac{1}{y}$ n'est pas intégrable à l'infini, la définition de $D_{ \pm}^{\gamma}$ doit être modifier pour $\gamma=0$. Donc on a la définition suivante:

$$
D_{ \pm}^{0} f(x)=-\int_{0}^{+\infty} \frac{f(x \pm y)-f(x) \mathbb{1}_{] 0,1}(y)}{y} d y
$$

pour $f \in \mathcal{C}^{\beta} \cap \mathrm{L}^{1}(\mathbb{R}), \beta>0$.

On note aussi $D^{0}:=D_{+}^{0}-D_{-}^{0}$ c'est la transformation de Hilbert modulo (le facteur $\frac{1}{\pi}$ ), nous renvoyons le lecteur à $[\mathbf{2 5}],[\mathbf{2 6}]$.

La formule suivante connue sous le nom (Switchnig Identity) joue un rôle important dans les démonstrations des théorèmes de la Section 3.

Soit $0 \leq \gamma<1$ et on suppose que $f, g \in \mathcal{C}^{\beta} \cap \mathrm{L}^{1}(\mathbb{R})$, alors pour $\beta>\gamma$ on a

$$
\int_{\mathbb{R}} f(x) D_{-}^{\gamma} g(x) d x=\int_{\mathbb{R}} g(x) D_{+}^{\gamma} f(x) d x .
$$

La dérivée fractionnaire a été étudiée par Ezawa et al. [9] pour des objectifs de la physique et qui apparaît naturellement dans certains théorèmes limites (Théorème 2.1 et Théorème 2.2 dans [26]). Elles apparaissent aussi de façon naturelle dans le calcul stochastique (cf. [11]) et la théorie spectrale des cordes vibrantes (cf. [2]). La fonctionnelle $H_{t}^{x}(-1-\gamma)$, $\left(0<\gamma<\frac{1}{2}\right)$ associée à la partie finie de Hadamard p.f. $\left(x_{+}^{-1-\gamma}\right)$ et la fonctionnelle $C_{t}^{x}$ associée à la valeur principale de Cauchy v. p. $\left(\frac{1}{x}\right)$ peuvent être obtenues moyennant la transformée de Hilbert et $D^{\gamma} L_{t}^{\bullet}$ : la dérivée fractionnaire d'ordre $\gamma$ par rapport à la variable $x$ du temps local brownien avec:

$$
H_{t}^{x}(-1-\gamma)=-\cos (\pi(1+\gamma)) D^{\gamma} L_{t}^{\bullet}(x)-\sin (\pi(1+\gamma)) \mathcal{H}\left(D^{\gamma} L_{t}^{\bullet}\right)(x),
$$


où $\mathcal{H}(f)$ est la transformée de Hilbert définie par

$$
\mathcal{H}(f)(s)=\int_{0}^{+\infty} \frac{f(s+u)-f(s-u)}{u} d u
$$

et $C_{t}^{x}$ a la représentation suivante:

$$
C_{t}^{x}=\mathcal{H}\left(L_{t}^{\bullet}\right)(x)
$$

(cf. [28], [24], [25], [26], [4] et [3]).

Avant de présenter notre améliorations citons d'abord la contribution de Yamada [25] qui a montré dans le cas du mouvement Brownien que le processus $\left(D^{\gamma} L_{t}^{\bullet}(x), t \geq 0, x \in \mathbb{R}\right)$ vérifie les conditions de Hölder suivantes: pour tout $T>0$ p.s.

$\forall 0<\beta<\frac{1}{2}-\gamma, \exists C>0$ telle que $\forall 0 \leq t, s \leq T, x \in \mathbb{R}$

$$
\left|D^{\gamma} L_{t}^{\bullet}(x)-D^{\gamma} L_{s}^{\bullet}(x)\right| \leq C|t-s|^{\beta},
$$

$\forall 0<\beta<\frac{1}{2}-\gamma, \exists C>0$ telle que $\forall 0 \leq t \leq T, x, y \in \mathbb{R}$ tel que $|x|,|y| \leq M, M>0$

$$
\left|D^{\gamma} L_{t}^{\bullet}(x)-D^{\gamma} L_{t}^{\bullet}(y)\right| \leq C|x-y|^{\beta} .
$$

Cette régularité de $D^{\gamma} L_{t}^{\bullet}$ entraîne celle de $H_{t}^{x}(-1-\gamma)$ :

Pour tous $T, M>0$ p.s.

$\forall 0<\beta<\frac{1}{2}-\gamma, \exists C>0$ telle que $\forall 0 \leq t, s \leq T,|x| \leq M$

$$
\left|H_{t}^{x}(-1-\gamma)-H_{s}^{x}(-1-\gamma)\right| \leq C|t-s|^{\beta},
$$

$\forall 0<\beta<\frac{1}{2}-\gamma, \exists C>0$ telle que $\forall 0 \leq t \leq T,|x|,|y| \leq M$

$$
\left|H_{t}^{x}(-1-\gamma)-H_{t}^{y}(-1-\gamma)\right| \leq C|x-y|^{\beta} .
$$

Remarque 3. Boufoussi et al. [7] ont donnés, dans le cas du mouvement Brownien également, des résultats plus fins que (4) et (5) plus précisément nous avons:

- Pour tous $t>0$ et $p<\infty$, la trajectoire $x \mapsto D^{\gamma} L_{t}^{\bullet}(x)$ vérifie p.s. la condition de Hölder d'indice $\frac{1}{2}-\gamma$ en norme $L^{p}(\mathbb{R})$.

- Pour tous $x \in \mathbb{R}, T>0$ et $0<\nu<\frac{1}{2}-\frac{\gamma}{2}$, la trajectoire $t \mapsto$ $D^{\gamma} L_{t}^{\bullet}(x)$ vérifie p.s. la condition de Hölder d'indice $\nu$ sur $[0, T]$.

Nous en déduisons en particulier que:

$\forall 0<\beta<\frac{1}{2}-\frac{\gamma}{2}$, et $0<\gamma<\frac{1}{2}, \exists C>0$ telle que $\forall 0 \leq t, s \leq T,|x| \leq M$

$$
\left|H_{t}^{x}(-1-\gamma)-H_{s}^{x}(-1-\gamma)\right| \leq C|t-s|^{\beta} \text {. }
$$


Pour plus de détails sur la dérivée fractionnaire nous renvoyons le lecteur à $[\mathbf{1 3}]$, [22] et $[\mathbf{2 1}]$.

Nous aurons, également, besoin d'un résultat de régularité de l'application $(t, x) \mapsto L_{t}^{x}$ par rapport aux variables espace temps.

Théorème 1. Soit $T>0$. Alors la condition suivante est vérifiée presque sûrement: pour tout $0<\beta_{1}<\frac{\alpha-1}{2 \alpha}$ et $0<\beta_{2}<\frac{\alpha-1}{2}$, il existe une constante $C>0$ telle que pour tous $(t, s) \in[0, T]^{2}$ et $(x, y) \in \mathbb{R}^{2}$ tel que $|x|,|y| \leq M$ on a

(6) $\quad|L(t, x)-L(t, y)-L(s, x)+L(s, y)| \leq C|t-s|^{\beta_{1}}|x-y|^{\beta_{2}}$.

Preuve: En appliquant la propriété de Markov pour $X$ en $s$ et la propriété de scaling on a pour tout $m \geq 1$ :

$$
\begin{aligned}
\mathbb{E} \mid L(t, x)-L & (t, y)-L(s, x)+\left.L(s, y)\right|^{2 m} \\
& =\mathbb{E}|L(t-s, x)-L(t-s, y)|^{2 m} \circ \theta_{s} \\
& =\mathbb{E}\left[\mathbb{E}|L(t-s, x)-L(t-s, y)|^{2 m} \circ \theta_{s} / X_{s}\right] \\
& =\int \mathbb{P}\left(X_{s} \in d z\right) \mathbb{E}|L(t-s, x-z)-L(t-s, y-z)|^{2 m} .
\end{aligned}
$$

Or d'après [19] on a: pour tout $m \geq 1$

$$
\mathbb{E}|L(t, x)-L(t, y)|^{2 m} \leq C(\alpha, m) t^{\frac{(\alpha-1)}{2 \alpha} 2 m}|x-y|^{\frac{\alpha-1}{2} 2 m}
$$

où $C(\alpha, m)$ est une constante qui dépend seulement de $\alpha$ et $m$. Par suite

$$
\begin{aligned}
\int \mathbb{P}\left(X_{s} \in d z\right) \mathbb{E} \mid & |L(t-s, x-z)-L(t-s, y-z)|^{2 m} \\
& \leq \int \mathbb{P}\left(X_{s} \in d z\right) C(\alpha, m)|t-s|^{\frac{(\alpha-1)}{2 \alpha} 2 m}|x-y|^{\frac{\alpha-1}{2} 2 m} \\
& \leq C(\alpha, m)|t-s|^{\frac{(\alpha-1)}{2 \alpha} 2 m}|x-y|^{\frac{\alpha-1}{2} 2 m} .
\end{aligned}
$$

D'où, si $\|\cdot\|_{2 m}$ désigne la norme $\left[\mathbb{E}[\cdot]^{2 m}\right]^{1 / 2 m}$, alors

(7) $\|L(t, x)-L(t, y)-L(s, x)+L(s, y)\|_{2 m}$

$$
\leq C(\alpha, m)|t-s|^{\frac{(\alpha-1)}{2 \alpha}}|x-y|^{\frac{\alpha-1}{2}} .
$$

Et d'après une version du théorème de Kolmogorov dans l'espace $\mathcal{H}_{0}^{a, b}$ (l'espace hölderien à deux paramètres) voir le Théorème 1.7 dans [6]), nous avons la conclusion du théorème. 
En utilisant les mêmes techniques que Yamada [25] (voir aussi [10, p. 319]) il est facile de prouver le corollaire suivant.

Corollaire 1. Soit $T$ un nombre réel strictement positif. $\forall 0<\gamma<\frac{\alpha-1}{2}, \exists C>0$ telle que $\forall 0 \leq t, s \leq T,|x| \leq M$,

$$
\left|D_{ \pm}^{\gamma} L_{t}^{\bullet}(x)-D_{ \pm}^{\gamma} L_{s}^{\bullet}(x)\right| \leq C|t-s|^{\beta}, \quad \forall \beta<(\alpha-1) / \alpha-\gamma .
$$

$\forall 0<\gamma<\frac{\alpha-1}{2}, \exists C>0$ telle que $\forall 0 \leq t, s \leq T,|x|,|y| \leq M$,

$$
\left|D_{ \pm}^{\gamma} L_{t}^{\bullet}(x)-D_{ \pm}^{\gamma} L_{s}^{\bullet}(y)\right| \leq C|x-y|^{\beta}, \quad \forall \beta<(\alpha-1) / 2-\gamma .
$$

De plus $\left|D_{ \pm}^{\gamma} L_{t}^{\bullet}(x)\right|=O\left(|x|^{-1-\gamma}\right),|x| \rightarrow \infty$ uniformément en $t$.

Dans le théorème suivant nous présentons un résultat plus fin que celui du corollaire ci-dessus et qui représente le résultat principal de cette section.

Théorème 2 (Régularité de la dérivée fractionnaire du temps local). Soient $T>0$ et $0 \leq \gamma<\frac{\alpha-1}{2}$, avec $\alpha$ l'indice du processus stable symétrique $X$. Alors presque sûrement pour tout $0<\lambda<\frac{\alpha-1}{\alpha}-\frac{\gamma}{\alpha}$, il existe une constante $0<C(\omega)<+\infty$ telle que pour tous $0 \leq t, s \leq T$ et $x \in \mathbb{R}$

$$
\left|D^{\gamma} L_{t}^{\bullet}(x)-D^{\gamma} L_{s}^{\bullet}(x)\right| \leq C(\omega)|t-s|^{\lambda} .
$$

Preuve: Nous faisons la démonstration pour $D_{+}^{\gamma}$ dans le cas de $\gamma>0$ (pour $\gamma=0$ nous utilisons les mêmes techniques que Fitzsimmons et Getoor [10, Lemme 2.12, p. 316]).

Soit $x \in \mathbb{R}$ et $0 \leq t, s \leq T$, d'après la définition de la dérivée fractionnaire on a

$$
\begin{aligned}
\mid D_{+}^{\gamma} L_{t}^{\bullet}(x)- & D_{+}^{\gamma} L_{s}^{\bullet}(x) \mid \\
= & \frac{1}{|\Gamma(-\gamma)|}\left|\int_{0}^{+\infty} \frac{L_{t}^{x+u}-L_{t}^{x}}{u^{1+\gamma}} d u-\int_{0}^{+\infty} \frac{L_{s}^{x+u}-L_{s}^{x}}{u^{1+\gamma}} d u\right| \\
\leq & \frac{1}{|\Gamma(-\gamma)|} \int_{0}^{+\infty} \frac{\left|L_{t}^{x+u}-L_{s}^{x+u}-L_{t}^{x}+L_{s}^{x}\right|}{u^{1+\gamma}} d u \\
\leq & \frac{1}{|\Gamma(-\gamma)|} \int_{0}^{b} \frac{\left|L_{t}^{x+u}-L_{s}^{x+u}-L_{t}^{x}+L_{s}^{x}\right|}{u^{1+\gamma}} d u \\
& +\frac{1}{|\Gamma(-\gamma)|} \int_{b}^{+\infty} \frac{\left|L_{t}^{x+u}-L_{s}^{x+u}-L_{t}^{x}+L_{s}^{x}\right|}{u^{1+\gamma}} d u \\
= & : I_{1}+I_{2} .
\end{aligned}
$$


Majoration de $I_{1}$ : En vertu de la condition de Hölder (6), du temps local en $t$ et $x$, nous avons

$$
I_{1} \leq \frac{C}{|\Gamma(-\gamma)|} \int_{0}^{b}|t-s|^{\beta_{1}} u^{\beta_{2}-1-\gamma} d u
$$

avec $\beta_{1}<(\alpha-1) / 2 \alpha$ et $\beta_{2}<(\alpha-1) / 2$.

Par conséquent

$$
I_{1} \leq \frac{C}{|\Gamma(-\gamma)|} \frac{b^{\beta_{2}-\gamma}}{\beta_{2}-\gamma}|t-s|^{\beta_{1}}, \text { avec } \gamma<\beta_{2} .
$$

Majoration de $I_{2}$ : Nous savons d'après (2) qu'il existe une variable aléatoire $C(\omega)$ finie telle que

$$
\left|L_{t}^{x}-L_{s}^{x}\right| \leq C(\omega)|t-s|^{\beta}
$$

où $\beta<\frac{\alpha-1}{\alpha}$, par suite

d'où

$$
I_{2} \leq \frac{2 C(\omega)}{|\Gamma(-\gamma)|} \int_{b}^{+\infty} \frac{|t-s|^{\beta}}{u^{1+\gamma}} d u=\frac{2 C(\omega)}{|\Gamma(-\gamma)|} \frac{b^{-\gamma}}{\gamma}|t-s|^{\beta},
$$

$$
I_{1}+I_{2} \leq \frac{2 C(\omega)}{|\Gamma(-\gamma)|} \frac{b^{\beta_{2}-\gamma}}{\beta_{2}-\gamma}|t-s|^{\beta_{1}}+\frac{2 C(\omega)}{|\Gamma(-\gamma)|} \frac{b^{-\gamma}}{\gamma}|t-s|^{\beta} ;
$$

en choisissant $b=|t-s|^{\frac{\beta-\beta_{1}}{\beta_{2}}}$, on obtient

$$
\left|D_{+}^{\gamma} L_{t}^{\bullet}(x)-D_{+}^{\gamma} L_{s}^{\bullet}(x)\right| \leq C(\omega)|t-s|^{\beta\left(1-\frac{\gamma}{\beta_{2}}\right)+\beta_{1} \frac{\gamma}{\beta_{2}}},
$$

par conséquent pour tout $0<\lambda<\frac{\alpha-1}{\alpha}-\frac{\gamma}{\alpha}$, on peut trouver $\gamma<\beta_{2}<$ $\frac{\alpha-1}{2} \leq \frac{1}{2}, 0<\beta<\frac{\alpha-1}{\alpha}$ et $0<\beta_{1}<\frac{\alpha-1}{2 \alpha}$ tel que $\lambda=\beta\left(1-\frac{\gamma}{\beta_{2}}\right)+\beta_{1} \frac{\gamma}{\beta_{2}}$.

Remarque 4. Ce résultat étend celui de [7] aux processus stable symétrique et donne une amélioration à celui de [25].

Le lemme suivant sera utile dans les preuves des théorèmes limites.

Lemme 1. Soient $T>0$ et $0 \leq \gamma<\frac{\alpha-1}{2}$, avec $\alpha$ l'indice du processus stable symétrique $X$ et $\left\{L_{t}^{x},(t, x) \in \mathbb{R}^{+} \times \mathbb{R}\right\}$ son temps local. Alors pour tous $0 \leq t, s \leq T, x \in \mathbb{R}$, et $m \geq 1$

$$
\left\|D^{\gamma} L_{t}^{\bullet}(x)-D^{\gamma} L_{s}^{\bullet}(x)\right\|_{2 m} \leq C|t-s|^{\frac{\alpha-1}{\alpha}-\frac{\gamma}{\alpha}}
$$

où la constante $C$ dépend seulement de $\alpha, m$ et $\gamma$. 
Preuve: Par définition de $D^{\gamma}$ nous avons

$$
\begin{aligned}
\left|D_{+}^{\gamma} L_{t}^{\bullet}(x)-D_{+}^{\gamma} L_{s}^{\bullet}(x)\right| \leq & \frac{1}{|\Gamma(-\gamma)|} \int_{0}^{b} \frac{\left|L_{t}^{x+u}-L_{s}^{x+u}-L_{t}^{x}+L_{s}^{x}\right|}{u^{1+\gamma}} d u \\
& +\frac{1}{|\Gamma(-\gamma)|} \int_{b}^{+\infty} \frac{\left|L_{t}^{x+u}-L_{s}^{x+u}-L_{t}^{x}+L_{s}^{x}\right|}{u^{1+\gamma}} d u
\end{aligned}
$$

ce qui entraîne

$$
\begin{aligned}
\| D_{+}^{\gamma} L_{t}^{\bullet}(x)- & D_{+}^{\gamma} L_{s}^{\bullet}(x) \|_{2 m} \\
\leq & \frac{1}{|\Gamma(-\gamma)|} \int_{0}^{b} \frac{\left\|L_{t}^{x+u}-L_{s}^{x+u}-L_{t}^{x}+L_{s}^{x}\right\|_{2 m}}{u^{1+\gamma}} d u \\
& +\frac{1}{|\Gamma(-\gamma)|} \int_{b}^{+\infty} \frac{\left\|L_{t}^{x+u}-L_{s}^{x+u}-L_{t}^{x}+L_{s}^{x}\right\|_{2 m}}{u^{1+\gamma}} d u \\
= & : J_{1}+J_{2} .
\end{aligned}
$$

En utilisant respectivement les inégalités (7) et (2) on obtient

$$
J_{1} \leq C_{1}(\alpha, m, \gamma)|t-s|^{\frac{\alpha-1}{2 \alpha}} b^{\frac{\alpha-1}{2}-\gamma},
$$

et

$$
J_{2} \leq C_{2}(\alpha, m, \gamma)|t-s|^{\frac{\alpha-1}{\alpha}} b^{-\gamma},
$$

en choisissant $b=|t-s|^{\frac{1}{\alpha}}$ on aura le résultat.

Remarque 5. Le Théorème 2 peut se déduire du lemme précédent à l'aide du critère de Kolmogorov.

\section{Théorèmes limites}

Dans cette section, nous présentons des théorèmes limites pour des processus de la forme

$$
\left.\frac{1}{\lambda^{p}} \int_{0}^{\lambda t} f\left(X_{s}\right) d s, \text { pour } p \in\right] \frac{\alpha-1}{2 \alpha}, \frac{\alpha-1}{\alpha}[
$$

où $\left\{X_{t}: t \geq 0\right\}$ un processus stable symétrique d'indice $1<\alpha \leq 2$ et $f$ est une dérivée fractionnaire d'une fonction $g \in \mathcal{C}^{\beta}$ (hölderienne d'ordre $\beta$ ) et à support compact, suivant la topologie hölderienne.

Pour $\alpha=2$, correspondant au cas du mouvement Brownien, le comportement asymptotique de la forme (9), suivant la fonction $f$, a été étudié par Yamada [26] et le cas $p \in] \frac{\alpha-1}{2 \alpha}, \frac{\alpha-1}{\alpha}[$ où $\alpha$ étant l'indice d'un 
procesus stable a été étudié par Fitzsimmons et Getoor [10] dans l'espace des fonctions continues.

\subsection{Critère de tension dans l'espace $\mathcal{C}_{0}^{\delta, 0}$.}

Pour étudier la convergence en loi des processus de la forme (9) pour la topologie hölderienne, nous utilisons le critère de tension que nous rappelons ci-dessous.

Lemme 2. Une suite de processus $\left\{\xi_{n}: n \geq 1\right\}$ converge en loi dans $\mathcal{C}_{0}^{\delta, 0}$ si et seulement si la suite des lois $\mathbb{P}_{n}=\mathbb{P} \circ \xi_{n}^{-1}$ des éléments aléatoires $\xi_{n}$ est tendue sur $\mathcal{C}_{0}^{\delta, 0}$ et on a la convergence des lois fini-dimensionnelles de $\xi_{n}$.

Théorème 3. Soit $\left\{\xi_{n}: n \geq 1\right\}$ une suite de processus nuls en zéro et vérifiant pour des constantes $a, b>0$ et $C>0$ et $\forall n \geq 1, \forall \lambda>0$,

$$
\mathbb{P}\left[\left|\xi_{n}(t)-\xi_{n}(s)\right|>\lambda\right] \leq C \lambda^{-a}|t-s|^{1+b},
$$

alors la suite des lois $\mathbb{P}_{n}$ des processus $\xi_{n}$ est tendue dans $\mathcal{C}_{0}^{\delta, 0}$ pour $0<\delta<b / a$.

La preuve du Lemme 2 se trouve dans [12] et celle du Théorème 3 dans $[\mathbf{1 7}]$.

Remarque 6. En pratique ce théorème dû à Lamperti [18] est utilisé sous sa version des moments:

$$
\sup _{n} \mathbb{E}\left|\xi_{n}(t)-\xi_{n}(s)\right|^{a} \leq C|t-s|^{1+b} .
$$

Nous présentons alors le résultat principal de cette section.

Théorème 4. Soit $X=\left\{X_{t}: t \geq 0\right\}$ un processus stable symétrique à valeur dans $\mathbb{R}$, d'indice $1<\alpha \leq 2$. Soit $0<\gamma<\frac{\alpha-1}{2}$ et on suppose que $f=D_{+}^{\gamma} g$, où $g \in \mathcal{C}^{\beta}$ et à support compact pour un certain $\beta$ tel que $0<\gamma<\beta<\frac{\alpha-1}{2}$ alors,

$$
\left\{\frac{1}{n^{1-\frac{1+\gamma}{\alpha}}} \int_{0}^{n t} f\left(X_{s}\right) d s\right\}_{t \geq 0} \stackrel{\mathcal{L}}{\longrightarrow}_{n \rightarrow+\infty}\left\{\left[\int_{\mathbb{R}} g(x) d x\right] D_{-}^{\gamma} L_{t}^{\bullet}(0)\right\}_{t \geq 0}
$$

et cette convergence a eu lieu dans l'espace de Hölder $\mathcal{C}_{0}^{\delta}$ avec $\delta<\frac{\alpha-1}{\alpha}-$ $\frac{\gamma}{\alpha}$.

Preuve: On fait la démonstration dans $\mathcal{C}_{0}^{\delta, 0}$ (car la convergence dans $\mathcal{C}_{0}^{\delta, 0}$ implique la convergence dans $\mathcal{C}_{0}^{\delta}$ ). 
Il est facile de verifier que la trajectoire

$$
t \longmapsto A_{t}^{n}=\frac{1}{n^{1-\frac{1+\gamma}{\alpha}}} \int_{0}^{n t} f\left(X_{s}\right) d s
$$

appartient p.s. à $\mathcal{C}_{0}^{\delta, 0}$ avec $\delta<\frac{\alpha-1}{\alpha}-\frac{\gamma}{\alpha}$. Or d'après [10] $\left\{A_{t}^{n}: t \geq 0\right\}$ converge en loi vers le processus $\left\{\left(\int_{\mathbb{R}} g(x) d x\right) D_{-}^{\gamma} L_{t}^{\bullet}(0)\right\}_{t \geq 0}$ dans l'espace des fonctions continues ce qui implique la convergence des lois finidimensionnelles. D'après le Lemme 2 il reste à montrer la tension. Pour le faire nous utilisons le critère de Lamperti [18, Remarque 6].

Soit $m \geq 1$, en utilisant (1) et (3) nous aurons

$$
\begin{aligned}
\mathbb{E}\left|A_{t}^{n}-A_{s}^{n}\right|^{2 m} & =\mathbb{E}\left|\frac{1}{n^{1-\frac{1+\gamma}{\alpha}}}\left(\int_{0}^{n t} f\left(X_{u}\right) d u-\int_{0}^{n s} f\left(X_{u}\right) d u\right)\right|^{2 m} \\
& =n^{2 m \frac{\gamma}{\alpha}} \mathbb{E}\left|\int_{\mathbb{R}} f(x) L_{t}^{x / n^{\frac{1}{\alpha}}} d x-\int_{\mathbb{R}} f(x) L_{s}^{x / n^{\frac{1}{\alpha}}} d x\right|^{2 m} \\
& =n^{2 m \frac{\gamma}{\alpha}} \mathbb{E}\left|\int_{\mathbb{R}} D_{+}^{\gamma} g(x)\left(L_{t}^{x / n^{\frac{1}{\alpha}}}-L_{s}^{x / n^{\frac{1}{\alpha}}}\right) d x\right|^{2 m} \\
& =n^{2 m \frac{\gamma}{\alpha}} \mathbb{E}\left|\int_{\mathbb{R}} g(x)\left(D_{-}^{\gamma} L_{t}^{\bullet / n^{\frac{1}{\alpha}}}(x)-D_{-}^{\gamma} L_{s}^{\bullet / n^{\frac{1}{\alpha}}}(x)\right) d x\right|^{2 m} .
\end{aligned}
$$

Or (si $h: \mathbb{R} \rightarrow \mathbb{R}$ et $a>0$ on note par $h_{a}$ la fonction $x \mapsto h(a x)$ alors $\left.D_{ \pm}^{\gamma}\left(h_{a}\right)=a^{\gamma}\left(D_{ \pm}^{\gamma} h\right)_{a}\right)$ par suite

$\mathbb{E}\left|A_{t}^{n}-A_{s}^{n}\right|^{2 m}$

$$
\begin{aligned}
& =\mathbb{E}\left|\int_{\mathbb{R}} g(x)\left(D_{-}^{\gamma} L_{t}^{\bullet}\left(x n^{\frac{-1}{\alpha}}\right)-D_{-}^{\gamma} L_{s}^{\bullet}\left(x n^{\frac{-1}{\alpha}}\right)\right) d x\right|^{2 m} \\
& \leq \mathbb{E}\left[\int_{K}|g(x)|^{m^{\prime}} d x\right]^{\frac{2 m}{m^{\prime}}} \int_{K}\left|D_{-}^{\gamma} L_{t}^{\bullet}\left(x n^{\frac{-1}{\alpha}}\right)-D_{-}^{\gamma} L_{s}^{\bullet}\left(x n^{\frac{-1}{\alpha}}\right)\right|^{2 m} d x
\end{aligned}
$$

avec $\frac{1}{2 m}+\frac{1}{m^{\prime}}=1$ et $K=\operatorname{supp}(g)$, par conséquent

$$
\begin{aligned}
\mathbb{E}\left|A_{t}^{n}-A_{s}^{n}\right|^{2 m} & \leq C \int_{K} \mathbb{E}\left|D_{-}^{\gamma} L_{t}^{\bullet}\left(x n^{\frac{-1}{\alpha}}\right)-D_{-}^{\gamma} L_{s}^{\bullet}\left(x n^{\frac{-1}{\alpha}}\right)\right|^{2 m} d x \\
& \leq C|t-s|^{\left(\frac{\alpha-1}{\alpha}-\frac{\gamma}{\alpha}\right) 2 m} \text { d'après (8). }
\end{aligned}
$$

D'où $\left(A_{t}^{n}\right)_{n}$ est tendue dans $\mathcal{C}_{0}^{\delta, 0}$ avec $\delta<\frac{\alpha-1}{\alpha}-\frac{\gamma}{\alpha}$. 
Théorème 5. Soit $X=\left\{X_{t}: t \geq 0\right\}$ un processus stable symétrique d'indice $1<\alpha \leq 2$. On suppose que $f=D_{+}^{0} g$ où $g \in \mathcal{C}^{\beta}$ à support compact pour $\beta>0$. Alors

i) $\left\{\frac{1}{n^{\frac{\alpha-1}{\alpha}} \log (n)} \int_{0}^{n t} f\left(X_{s}\right) d s\right\}_{t>0}$ converge en loi lorsque $n$ tend vers $+\infty$ vers le processus $\left\{-\alpha^{-1}\left(\int_{\mathbb{R}} g(x) d x\right) L_{t}^{0}\right\}_{t>0}$.

ii) $\left\{\frac{1}{n \frac{\alpha-1}{\alpha}} \int_{0}^{n t}\left(f\left(X_{s}\right)+\alpha^{-1} \log (n) g\left(X_{s}\right)\right) d s\right\}_{t \geq 0}$ converge en loi lorsque $n$ tend vers $+\infty$ vers le processus $\left\{\left(\int_{\mathbb{R}} g(x) d x\right) D_{-}^{0} L_{t}^{\bullet}(0)\right\}_{t>0}$. Ces convergences ont eux lieu dans l'espace de Hölder $\overline{\mathcal{C}}_{0}^{\delta}$ avec $\delta<\frac{\alpha-1}{\alpha}$.

Preuve: Pour i) on pose

$$
A_{t}^{n}=\frac{1}{n^{1-\frac{1}{\alpha}} \log (n)} \int_{0}^{n t} f\left(X_{s}\right) d s
$$

par les mêmes arguments de la démonstration du Théorème 4 et le fait que $\left(\right.$ si $h: \mathbb{R} \rightarrow \mathbb{R}$ et $a>0$ on note par $h_{a}$ la fonction $x \mapsto h(a x)$ alors $\left.D_{ \pm}^{0}\left(h_{a}\right)=\left(D_{ \pm}^{0} h\right)_{a}+h_{a} \log a\right)$ on a

$$
\begin{aligned}
A_{t}^{n} & \stackrel{\mathcal{L}}{=} \frac{1}{\log (n)} \int_{\mathbb{R}} g(x) D_{-}^{0}\left(L_{t}^{\bullet}\left(x n^{\frac{-1}{\alpha}}\right)\right) d x-\alpha^{-1} \int_{\mathbb{R}} g(x) L_{t}^{x n^{\frac{-1}{\alpha}}} d x \\
& =: B_{t}^{n}-F_{t}^{n} .
\end{aligned}
$$

Montrons que $B_{t}^{n}$ converge vers 0 dans $\mathcal{C}_{0}^{\delta, 0}$ lorsque $n$ tend vers $+\infty$ avec $\delta<\frac{\alpha-1}{\alpha}$.

Il est claire que $B_{t}^{n} \in \mathcal{C}_{0}^{\delta, 0}$, car

$$
\begin{aligned}
& \lim _{\eta \rightarrow 0} \omega_{\delta}\left(B_{t}^{n}, \eta\right) \\
= & \lim _{\eta \rightarrow 0} \sup _{0<|t-s|<\eta} \frac{\left|B_{t}^{n}-B_{s}^{n}\right|}{|t-s|^{\delta}} \\
= & \lim _{\eta \rightarrow 0} \sup _{0<|t-s|<\eta} \frac{1}{\log (n)} \frac{\left|\int_{\mathbb{R}} g(x)\left[D_{-}^{0}\left(L_{t}^{\bullet}\left(x n^{\frac{-1}{\alpha}}\right)\right)-D_{-}^{0}\left(L_{s}^{\bullet}\left(x n^{\frac{-1}{\alpha}}\right)\right)\right] d x\right|}{|t-s|^{\delta}} \\
\leq & \lim _{\eta \rightarrow 0} \sup _{0<|t-s|<\eta} \frac{C}{\log (n)}|t-s|^{\lambda-\delta}, \quad \text { avec } 0<\lambda<\frac{\alpha-1}{\alpha} \\
\leq & \lim _{\eta \rightarrow 0} \frac{C}{\log (n)} \eta^{\frac{\alpha-1}{\alpha}-\varepsilon-\delta}=0, \quad \text { pour } \delta<\frac{\alpha-1}{\alpha} \leq \frac{1}{2} .
\end{aligned}
$$


Par conséquent

$$
\begin{aligned}
\omega_{\delta}\left(B_{t}^{n}, 1\right) & =\sup _{0<|t-s|<1} \frac{\left|B_{t}^{n}-B_{s}^{n}\right|}{|t-s|^{\delta}} \\
& =\sup _{0<|t-s|<1} \frac{1}{\log (n)} \frac{\left|\int_{\mathbb{R}} g(x)\left(D_{-}^{0} L_{t}^{\bullet}\left(x n^{-\frac{1}{\alpha}}\right)-D_{-}^{0} L_{s}^{\bullet}\left(x n^{-\frac{1}{\alpha}}\right)\right) d x\right|}{|t-s|^{\delta}} \\
& \leq \sup _{0<|t-s|<1} \frac{1}{\log (n)} \frac{\int_{\mathbb{R}}|g(x)|\left|D_{-}^{0} L_{t}^{\bullet}\left(x n^{-\frac{1}{\alpha}}\right)-D_{-}^{0} L_{s}^{\bullet}\left(x n^{-\frac{1}{\alpha}}\right)\right| d x}{|t-s|^{\delta}} \\
& \leq \sup _{0<|t-s|<1} \frac{C}{\log (n)}|t-s|^{\lambda-\delta}=: I_{n} \text { pour } 0<\lambda<\frac{\alpha-1}{\alpha} .
\end{aligned}
$$

D'où $I_{n} \longrightarrow_{n \rightarrow+\infty} 0$ pour $\delta<\frac{\alpha-1}{\alpha}$.

Montrons maintenant que $F_{t}^{n}$ converge en loi vers $\alpha^{-1}\left(\int_{\mathbb{R}} g(x) d x\right) L_{t}^{0}$ $\operatorname{dans} \mathcal{C}_{0}^{\delta, 0}$.

Nous savons que

$$
\int_{\mathbb{R}} g(x) L_{t}^{x n^{-\frac{1}{\alpha}}} d x \stackrel{\mathcal{L}}{\longrightarrow}_{n \rightarrow+\infty}\left(\int_{\mathbb{R}} g(x) d x\right) L_{t}^{0}
$$

dans l'espace des fonctions continues. Il reste à montrer la tension. Soit $m \geq 1$

$$
\begin{aligned}
\mathbb{E}\left|F_{t}^{n}-F_{s}^{n}\right|^{2 m} & =\alpha^{-2 m} \mathbb{E}\left|\int_{\mathbb{R}} g(x)\left(L_{t}^{x n^{-\frac{1}{\alpha}}}-L_{s}^{x n^{-\frac{1}{\alpha}}}\right) d x\right|^{2 m} \\
& \leq \alpha^{-2 m} \mathbb{E}\left(\int_{K}|g(x)|^{m^{\prime}} d x\right)^{\frac{2 m}{m^{\prime}}} \int_{K}\left|L_{t}^{x n^{-\frac{1}{\alpha}}}-L_{s}^{x n^{-\frac{1}{\alpha}}}\right|^{2 m} d x \\
& \leq C|t-s|^{\frac{\alpha-1}{\alpha} 2 m} \text { d'après (8) }
\end{aligned}
$$

et $\left(F_{t}^{n}\right)_{n \geq 0}$ est tendue dans $\mathcal{C}_{0}^{\delta, 0}$ pour tout $\delta<\frac{\alpha-1}{\alpha}$.

Pour ii) de la même façon nous avons

$$
\frac{1}{n^{\frac{\alpha-1}{\alpha}}} \int_{0}^{n t}\left(f\left(X_{s}\right)+\alpha^{-1} \log (n) g\left(X_{s}\right)\right) d s \stackrel{\mathcal{L}}{=} \int_{\mathbb{R}} g(x) D_{-}^{0} L_{t}^{\bullet}\left(x n^{-\frac{1}{\alpha}}\right) d x .
$$

Or d'après [10]

$$
\int_{\mathbb{R}} g(x) D_{-}^{0} L_{t}^{\bullet}\left(x n^{-\frac{1}{\alpha}}\right) d x \longrightarrow_{n \rightarrow+\infty}\left(\int_{\mathbb{R}} g(x) d x\right) D_{-}^{0} L_{t}^{\bullet}(0)
$$

dans l'espace des fonctions continues. 
Il suffit, alors, de montrer la tension dans l'espace $\mathcal{C}_{0}^{\delta, 0}$. Posons

$$
E_{t}^{n}:=\int_{\mathbb{R}} g(x) D_{-}^{0} L_{t}^{\bullet}\left(x n^{-\frac{1}{\alpha}}\right) d x .
$$

Soit $m \geq 1$ nous avons

$$
\begin{aligned}
\mathbb{E} & \left|E_{t}^{n}-E_{s}^{n}\right|^{2 m} \\
& =\mathbb{E}\left|\int_{\mathbb{R}} g(x)\left(D_{-}^{0} L_{t}^{\bullet}\left(x n^{-\frac{1}{\alpha}}\right)-D_{-}^{0} L_{s}^{\bullet}\left(x n^{-\frac{1}{\alpha}}\right)\right) d x\right|^{2 m} \\
& \leq\left(\int_{K}|g(x)|^{m^{\prime}} d x\right)^{\frac{2 m}{m^{\prime}}} \mathbb{E} \int_{K}\left|D_{-}^{0} L_{t}^{\bullet}\left(x n^{-\frac{1}{\alpha}}\right)-D_{-}^{0} L_{s}^{\bullet}\left(x n^{-\frac{1}{\alpha}}\right)\right|^{2 m} d x \\
& \leq C|t-s|^{\frac{\alpha-1}{\alpha} 2 m}
\end{aligned}
$$

donc $\left(E_{t}^{n}\right)_{n \geq 0}$ est tendue dans $\mathcal{C}_{0}^{\delta, 0}$ avec $\delta<\frac{\alpha-1}{\alpha}$.

\section{Références}

[1] M. T. BARLOW, Necessary and sufficient conditions for the continuity of local time of Lévy processes, Ann. Probab. 16(4) (1988), 1389-1427.

[2] J. Bertoin, Applications de la théorie spectrale des cordes vibrantes aux fonctionnelles additives principales d'un brownien réfléchi, Ann. Inst. H. Poincaré Probab. Statist. 25(3) (1989), 307-323.

[3] J. Bertoin, Complements on the Hilbert transform and the fractional derivative of brownian local times, J. Math. Kyoto Univ. 30(4) (1990), 651-670.

[4] P. Biane ET M. Yor, Valeurs principales associées aux temps locaux browniens, Bull. Sci. Math. (2) 111(1) (1987), 23-101.

[5] R. M. Blumenthal et R. K. Getoon, "Markov processes and potential theory", Pure and Applied Mathematics 29, Academic Press, New York, 1968.

[6] B. Boufoussi, Espaces de Besov, caractérisation et applications, Thèse de l'Université Henri Poincaré, Nancy I, France (1994).

[7] B. Boufoussi, M. Eddahbi et A. Kamont, Sur la dérivée fractionnaire du temps local brownien, Probab. Math. Statist. 17(2) (1997), 311-319.

[8] E. S. Boylan, Local times for a class of Markoff processes, Illinois J. Math. 8 (1964), 19-39. 
[9] H. Ezawa, J. R. Klauder et L. A. Shepp, Vestigial effects of singular potentials in diffusion theory and quantum mechanics, $J$. Mathematical Phys. 16 (1975), 783-799.

[10] P. J. Fitzsimmons et R. K. Getoor, Limit theorems and variation properties for fractional derivatives of the local time of a stable process, Ann. Inst. H. Poincaré Probab. Statist. 28(2) (1992), 311-333.

[11] M. Fukushima, A decomposition of additive functionals of finite energy, Nagoya Math. J. 74 (1979), 137-168.

[12] D. Hamadouche, Convergence de processus stochastique à trajectoires hölderiennes, Thèse de l'Université des Sciences et Technologie de Lille (1997).

[13] G. H. Hardy et J. E. Littlewood, Some properties of fractional integrals, I, Math. Z. 27 (1928), 567-606.

[14] K. Itô ET H. P. MCKeAN, "Diffusion processes and their sample paths", Die Grundlehren der Mathematischen Wissenschaften 125, Academic Press Inc., Publishers, New York, 1965.

[15] Y. Kasahara, Limit theorems of occuptation times for Markov processes, Publ. Res. Inst. Math. Sci. 12(3) (1976/77), 801-818.

[16] Y. Kasahara, Two limit theorems for occupation times of Markov processes, Japan. J. Math. (N.S.) 7(2) (1981), 291-300.

[17] G. Kerkyacharian et B. Roynette, Une démonstration simple des théorèmes de Kolmogorov, Donsker et Itô-Nisio, C. R. Acad. Sci. Paris Sér. I Math. 312(11) (1991), 877-882.

[18] J. LAmperti, On convergence of stochastic processes, Trans. Amer. Math. Soc. 104 (1962), 430-435.

[19] M. B. Marcus et J. Rosen, $p$-variation of the local times of symmetric stable processes and of Gaussian processes with stationary increments, Ann. Probab. 20(4) (1992), 1685-1713.

[20] S. NAKAO, Stochastic calculus for continuous additive functionals of zero energy, Z. Wahrsch. Verw. Gebiete 68(4) (1985), 557-578.

[21] S. G. Samko, A. A. Kilbas et O. I. Marichev, "Fractional integrals and derivatives. Theory and applications", Gordon and Breach Science Publishers, Yverdon, 1993.

[22] E. C. Titchmarsh, "Introduction to the theory of Fourier integrals", Second ed., Clarendon Press, Oxford, 1948.

[23] H. F. Trotter, A property of brownian motion paths, Illinois J. Math. 2 (1958), 425-433.

[24] T. YAmADA, On some representations concerning the stochastic integrals, Probab. Math. Statist. 4(2) (1984), 153-166. 
[25] T. YAMADA, On the fractional derivative of brownian local times, J. Math. Kyoto Univ. 25(1) (1985), 49-58.

[26] T. YAMADA, On some limit theorems for occupation times of onedimensional brownian motion and its continuous additive functionals locally of zero energy, J. Math. Kyoto Univ. 26(2) (1986), 309-322.

[27] T. Yamada, Principal values of brownian local times and their related topics, in "Itô's stochastic calculus and probability theory", Springer, Tokyo, 1996, pp. 413-422.

[28] M. Yor, Sur la transformée de Hilbert des temps locaux browniens, et une extension de la formule d'Itô, in "Seminar on Probability, XVI", Lecture Notes in Math. 920, Springer, Berlin, 1982, pp. 238-247.

M. Ait Ouahra:

Université Cadi Ayyad

Faculté des Sciences Semlalia

Département de Mathématiques

B.P.S 15, Marrakech

Maroc

E-mail address: ouahra@ucam.ac.ma

M. Eddahbi:

Université Cadi Ayyad

Faculté des Sciences et Techniques

Département de Mathématiques

B.P. 618, Marrakech

Maroc

E-mail address: eddahbi@fstg-marrakech.ac.ma

Primera versió rebuda el 7 de setembre de 2000, darrera versió rebuda el 8 de febrer de 2001. 